\section{AB0523 AN ANALYSIS OF THE FACTORS INFLUENCING THE BODY IMAGE DISTURBANCE WITH SYSTEMIC LUPUS ERYTHEMATOSUS LIVING IN CHINA}

Q. Zhao, Y. Cui, L. Li, S. Chen, C. Zhou, Z. Gu. Department of Rheumatology, Affiliated Hospital of Nantong University, Nantong, China

Background: Systemic Lupus Erythematosus (SLE) is a chronic, autoimmune rheumatic disease that can affect multiple systems/organs in the body and is characterized by relapses and remission, usually affects women in the reproductive age group. SLE may cause inflammation activity and damage in any of the organs, often resulting in decreased physical, emotional and social abilities, depression, pain, fatigue, visible or invisible changes in appearance. Recent studies have indicated that body image disturbance (BID) can lead to a variety of cognitive, emotional and behavioral changes. BID will cause the individual having a negative perception of the body, negative emotional experience and the corresponding behavior disorders.

Objectives: To explore the relationship between social and economic status, disease activity, mental state, self-esteem, quality of life and the essential BID with Systemic Lupus Erythematosus Living in China.

Methods: The investigation is from in October 2015 to December 2016 at the Affiliated Hospital of Nantong University. There are 109 cases of hospitalized patients, including 8 cases of male, female 101 cases, mean age is 35.3 \pm 12.2 . Scale including body image disorder scale (BID), respectively. Body image was assessed using the Body Image Disturbance Questionnaire (BIDQ) which contains seven scaled items scored from 0 (not affected) to 8 (extremely affected) pertaining to appearance-related concerns (BIDQ1); mental preoccupation (BIDQ2); emotional distress (BIDQ3); social, occupational, or functional impairment (BIDQ4); social life interference (BIDQ5) and educational, occupational, or other functional interferences (BIDQ6); and behavioural avoidance (BIDQ7). Systemic lupus erythematosus disease activity index (SLEDAI); the hospital anxiety and Depression Scale (HADS) in systemic lupus erythematosus; disease-specific HRQOL measure for adults with SLE (Lupus QOL), including health $(\mathrm{PH})$, pain $(\mathrm{PN})$, planning $(\mathrm{PL})$, intimate relationship $(\mathrm{IR})$, a burden to others (BV), emotional Health (EH), body image (BI), fatigue (F). Spearman's coefficient for nonparametric and Pearson's coefficient for parametric data were computed. Variables that were significant in univariate analysis were included in a multivariate linear regression model. All p-values less than 0.05 were considered statistically significant.

Results: SLE patients were most concerned about their body size $(33.38 \%)$ and skin $(32.23 \%), 55.34 \%$ SLE patients were more concerned about the change of body shape caused by disease. There was significant correlation between BID and disease status, psychological status and quality of life in patients with SLE. Physical health is correlated with BD1, Pain is correlated with BID1, BID4-7, Planning is correlated with BID2, BID4-7, Intimate relationships is correlated with BID4-7, Burden to others is correlated with BID3-7, Emotional health, fatigue are associated with BID3-7. Anxiety and depression also affect BID, anxiety is an important predictor of BIDQ1, BIDQ3-6, depression is a predictor of BIDQ2, BIDQ 3-6.

Conclusions: Patients with Systemic Lupus Erythematosus Living in China are much easier bear with BID, anxiety, depression, less quality of life will lead to BID, in view of the body image perspective, this study will help explore the influence factors of BID in patients with SLE.

Acknowledgements: The study was supported by The Natural Science Foundation of China (81401124)

Disclosure of Interest: None declared

DOI: 10.1136/annrheumdis-2017-eular.5284

\section{AB0524 TRANSLATION AND VALIDATION OF THE FATIGUE SEVERITY SCALE, THE PITTSBURGH SLEEP QUALITY INDEX AND THE MODIFIED HEALTH ASSESSMENT QUESTIONNAIRE INTO THE MALTESE LANGUAGE, IN A COHORT OF MALTESE PATIENTS WITH SYSTEMIC LUPUS ERYTHEMATOSUS}

\section{R. Magro ${ }^{1}$, A.A. Borg ${ }^{1}$, L. Camilleri ${ }^{2} .{ }^{1}$ Rheumatology, Mater Dei Hospital;} ${ }^{2}$ Statistics and Operations Research Department, University of Malta, Msida, Malta

Background: The Fatigue Severity Scale (FSS), Pittsburgh Sleep Quality Index (PSQI) and Modified Health Assessment Questionnaire (mHAQ) are validated questionnaires that measure fatigue, sleep quality and function respectively. Research in the Maltese population, on systemic lupus erythematosus (SLE) requiring the use of these questionnaires, necessitates their translation and validation into the Maltese language.

Objectives: The aim of the study is to translate, validate and perform crosscultural adaptation of the FSS, PSQI and $\mathrm{mHAQ}$ into the Maltese language, according to the recommended methodology. ${ }^{1}$

Methods: The original instruments were translated into Maltese by two translators whose mother language is Maltese. One of the translators had a medical background and was knowledgeable on the concepts being examined in the questionnaires; while the other did not. The two translated versions of the instruments were then compared and the discrepancies were resolved by reaching a consensus on the best Maltese version that reflects the underlying meaning of the original English version. The preliminary initial Maltese translations were then given to two other translators for back translation into English. English was the native language of these translators and they were totally blind to the original versions. The back translated English versions were then compared to the original English questionnaires and any discrepancies were highlighted. The equivalent statements in the Maltese translations were discussed and changes were made to reflect the original English versions more accurately. The pre-final Maltese translations were produced and pilot tested in a sample of 20 bilingual SLE patients to evaluate the clarity of the questionnaires. These 20 patients were also asked to fill in the original English version of the FSS, PSQI and $\mathrm{mHAQ}$, seven days after filling in the Maltese versions. Psychometric testing was carried out to assess reliability of the translation, internal consistency and validity. Results: Reliability of the translation of the FSS, PSQI and $\mathrm{mHAQ}$ into Maltese was analysed by using Kendall's tau test for statements having an ordinal scale and Pearson's correlation test for variables having a metric scale. The $p$ value for each statement in the FSS, PSQI and mHAQ was less than 0.05 showing that the reliability of the translated versions was satisfactory. Internal consistency of the Maltese translations was demonstrated using Cronbach's alpha. This was calculated to be 0.877 for the FSS, 0.859 for the PSQI, and 0.897 for the mHAQ. Validity of the Maltese translation of the FSS was assessed by its correlation with the visual analogue scale for fatigue using Pearson's Correlation test. Pearson's $R$ value was 0.809 and the $p$ value was $<0.001$ confirming a positive significant correlation.

Conclusions: This study has confirmed the reliability and internal consistency of the translated English versions of the FSS, PSQI and mHAQ into Maltese. Moreover the validity of the Maltese version of the FSS has been shown. The Maltese translations can be finalised and used for research purposes.

\section{References:}

[1] Beaton DE, Bombardier C, Guillemin F, Ferraz MB. Guidelines for the process of cross-cultural adaptation of self-report measures. Spine. 2000; 25(24): 3186-3191.

Disclosure of Interest: None declared

DOI: 10.1136/annrheumdis-2017-eular.220

\section{AB0525 CLINICAL AND RENAL HISTOLOGIAL CHARACTERISTICS OF PATIENTS WITH SYSTEMIC LUPUS ERYTHEMATOSUS WITH ANTI-SS-A AND ANTI-SS-B ANTIBODIES COMPARED TO THOSE WITHOUT}

R. Anan, Y. Kaneko, J. Kikuchi, M. Ushikubo, S. Saito, T. Takeuchi. Division of Rheumatology, Department of Internal Medicine, Keio University School of Medicine, Shinjuku-ku, Tokyo, Japan

Background: Systemic lupus erythematosus (SLE) is a chronic condition with a broad clinical spectrum, which can affect multiple organs and systems. Although the most featuristic autoantibodies are anti-DNA and anti-Sm antibodies, anti-SSA antibody and anti-SS-B antibody are detected in $30-40 \%$ and $7-45 \%$ of SLE patients, respectively. However, the clinical features of those positive patients are unclear.

Objectives: The aim of this study was to clarify the associations of anti-SS-A and anti-SS-B antibodies with the clinical features of SLE and histological findings of lupus nephritis (LN)

Methods: We enrolled consecutive Japanese patients with SLE who had visited Keio University Hospital from 2013 to 2015 and whose anti-SSA and anti-SSB antibodies were measured. The patients were divided according to the presence or absence of anti-SS-A and anti-SS-B antibodies, and their associations of the clinical features and renal biopsy histological findings were examined.

Results: A total of 253 patients were enrolled, 215 (84\%) were female, and the mean age was 55.2 years. Anti-SS-A antibody was detected in 152 cases $(69 \%)$, and anti-SS-B antibody in 40 cases $(18 \%)$. No difference was found in sex, age, the rates of anti-DNA and anti-Sm positivity between both the anti-SS-A positive group and the anti-SS-A negative group, and between the anti-SS-B positive group and the anti-SS-B negative group. While the frequencies of oral ulcers (38\% vs. $37 \%, p=0.83)$, arthritis ( $82 \%$ vs. $79 \%, p=0.58)$, serositis ( $23 \%$ vs. $22 \%, p=0.94$ ), neuropsychiatric involvement ( $14 \%$ vs. $10 \%, p=0.35), L N(51 \%$ vs. $63 \%, p=0.07)$, leukopenia ( $74 \%$ vs. $69 \%, p=0.44)$, and thrombocytopenia $(26 \%$ vs. $31 \%, p=0.44$ ) were not different between the anti-SS-A positive group and the anti-SS-A negative group,.mucocutaneous involvement ( $84 \%$ vs. $63 \%, p=0.002)$, alopecia ( $37 \%$ vs. $19 \%, p=0.002)$, lymphopenia ( $97 \%$ vs. $89 \%, p=0.020$ ) were significantly higher in the anti-SS-A positive group than the anti-SS-A negative group. The frequencies of neuropsychiatric involvement $(24 \%$ vs. $10 \%, p=0.015)$ was significantly higher in the anti-SS-B positive group than the anti-SS-B negative group. The period from the SLE onset to the LN onset was significantly shorter in the anti-SS-A positive group than the anti-SS-A negative group (4.0 years vs. 9.0 years, $p=0.005$ ), and in the anti-SS-B positive group than the anti-SS-B negative group ( 0.4 years vs. 6.8 years, $p=0.008)$. Focusing on the histological findings of LN patients, the anti-SS-A positive group had significantly lower rate of renal tubular atrophy $(8.0 \%$ vs. $16 \%, p=0.042)$ and interstitial fibrosis $(7.6 \%$ vs. $16 \%$, $\mathrm{p}=0.031$ ) than the anti-SS-A negative group. The anti-SS-B positive group had also significantly lower rate of renal tubular atrophy $(3.3 \%$ vs. $13 \%, p=0.048)$ and interstitial fibrosis $(2.1 \%$ vs. $13 \%, p=0.016)$. Finally, chronicity Index was significantly lower in the anti-SS-A positive group ( 2.37 vs. $4.14, p=0.002)$ and in the anti-SS-B positive group (1.17 vs. 3.33, $p=0.008$ ).

Conclusions: SLE patients positive for anti-SS-A and anti-SS-B antibodies had 
characteristics with frequent mucocutaneous involvement, alopecia, neuropsychiatric involvement, and lymphopenia. They should be followed carefully for early onset of LN.

Disclosure of Interest: None declared

DOI: 10.1136/annrheumdis-2017-eular.3964

\section{AB0526 B1 CD5+ LYMPHOCYTES IN SYSTEMIC LUPUS ERYTHEMATOSUS PATIENTS: RELATION TO DISEASE ACTIVITY}

S.I. Nasef ${ }^{1}$, H.H. Omar ${ }^{2}$, H.H. Omar ${ }^{3}$, M.S. Ghaly ${ }^{1} .{ }^{1}$ Physical Medicine, Rheumatology and Rehabilitation; ${ }^{2}$ Clinical Pathology; ${ }^{3}$ Internal Medicine, Faculty of Medicine, Suez Canal University, Ismailia, Egypt

Background: B cells are essential players in the pathogenesis of Systemic lupus erythematosus (SLE). Membrane CD5 elevates the threshold of B cell receptor mediated responses, and thus prevents the release of antibodies. So, misguided signalling through CD5 could lead to autoimmunity. Hence, CD5 ${ }^{+}$ $B$ cells were considered to play a paradoxical role in preventing rather than inducing autoimmunity. This challenging view differs from the old interpretation that elevated levels of B1 CD5+ cells in SLE patients represent a direct source of autoantibodies responsible for organ damage.

The clinical implications of this new concept for the role of $\mathrm{B} 1 \mathrm{CDF}^{+}$cells in SLE have not been fully addressed yet and there is no consensus agreement about the proportions of B1 CD5+ cells in SLE patients. Moreover, the relation of B1 $\mathrm{CD}^{+}$cells to disease activity and organ damage is not sufficiently studied.

Objectives: To assess the expression of $\mathrm{B}^{\mathrm{CD} 5^{+}}$cells in SLE patients and to evaluate their relationship with disease activity and organ damage.

Methods: We recruited 100 SLE patients and 100 healthy control subjects. Based on SLE disease activity index (SLEDAI), patients were divided into two groups: active SLE $(n=50)$ and inactive SLE $(n=50)$. SLE was active when SLEDAI was $>4$. The expression of $\left(\mathrm{CD}^{+} \mathrm{CD} 2 \mathrm{O}^{+}\right) \mathrm{B} 1$ cells was evaluated using flow cytometry. Lymphocytes were gated depending on both side and forward scatter. From the gated lymphocytes, B1a cells were identified double positive cells for CD20 and CD5. Percentage and absolute numbers of $\mathrm{CD}_{2} \mathrm{O}^{+} \mathrm{CD}^{+}$(B1a cells) and their mean fluorescence intensity (MFI) were measured. The histogram of CD5 expression was used to assess its expression on CD20 cells (figure 1).

Laboratory work included CBC, ESR, CRP, Serum creatinine, Protein/creatinine ratio, Urine analysis, 24 hour protein collection in urine, Complement levels, (Anti-dsDNA) and (ANA).

Results: Mean age of patients was $31.3 \pm 8.8$ years. Females constituted $94 \%$ $(n=94)$ of patients. Mean disease duration was $5.28 \pm 4.8$ years. Mean SLEDAI was $10.28 \pm 5.16$.

The proportions of $\left(\mathrm{CD}^{+} \mathrm{CD} 20^{+}\right) \mathrm{B} 1$ cells were significantly lower in SLE patients versus controls $(5.9 \pm 4.4 \%$ vs $20.2 \pm 4 \%, p \leq 0.001)$. Similarly, the absolute numbers of $\left(\mathrm{CDF}^{+} \mathrm{CD} 2 \mathrm{O}^{+}\right) \mathrm{B} 1$ cells $\left(\mathrm{cell} / \mathrm{mm}^{3}\right)$ were significantly lower in SLE patients versus controls $(100.2 \pm 103.4$ vs $557.6 \pm 163.3, p<0.001)$

The expression of $\left(\mathrm{CD}^{+} \mathrm{CD} 2 \mathrm{O}^{+}\right) \mathrm{B} 1$ cells was decreased in active SLE patients $(4.5 \pm 3.8 \%)$ in comparison to inactive patients $(7.3 \pm 4.7 \%)(p=0.027)$. B1 $\left(\mathrm{CDF}^{+} \mathrm{CD}^{2} \mathrm{O}^{+}\right)$absolute cell number $\left(\mathrm{cell} / \mathrm{mm}^{3}\right)$ was significantly lower in active SLE patients $(71.4 \pm 82.9)$ compared to inactive ones $(129.0 \pm 115.1)(P=0.047)$.
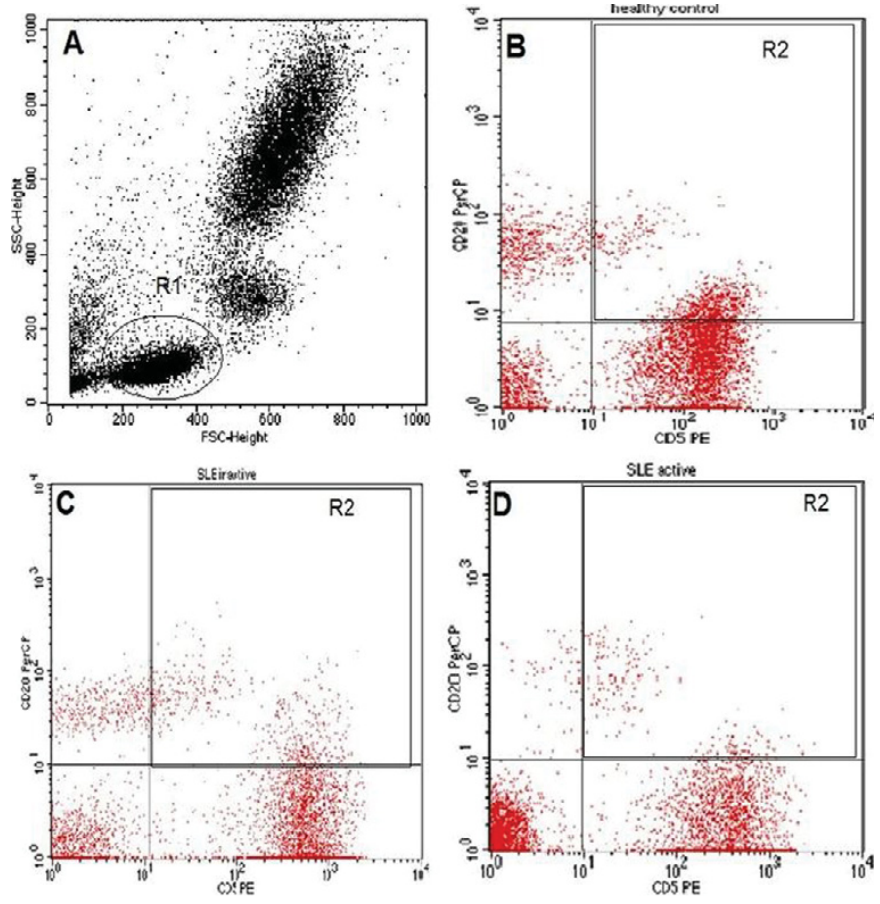

Figure 1
$\mathrm{MFI}$ of $\mathrm{CD}^{+} / \mathrm{CD}^{2} 0^{+}$was significantly decreased in SLE patients compared to healthy control $(146.9 \pm 109$ vs $196 \pm 48, P=0.033)$. B1 cells $\left(C D 5^{+} C D 20^{+}\right)$ correlated positively with $\mathrm{C} 3(\mathrm{r}=0.322, p=0.022)$ and $\mathrm{C} 4(\mathrm{r}=0.307, p=0.030)$. No correlation was found between $\left(\mathrm{CDF}^{+} \mathrm{CD} 2 \mathrm{O}^{+}\right) \mathrm{B} 1$ cells and disease duration, autoantibodies or any specific system or organ damage.

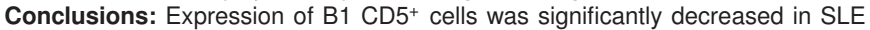
patients. Decreased $\mathrm{B} 1 \mathrm{CD}^{+}$cells expression was associated with higher disease activity. $\mathrm{B} 1 \mathrm{CD}^{+}$correlated positively with complement levels. These findings denote that CD5 expression on B cells may play a regulatory role in SLE pathogenesis and decrease occurrence of flares.

Disclosure of Interest: None declared

DOI: 10.1136/annrheumdis-2017-eular.2006

\section{AB0527 PROGRANULIN AND INSULIN-LIKE GROWTH FACTOR-2 AS BIOMARKERS FOR DISEASE ACTIVITY AND PATHOLOGICAL CHANGES IN LUPUS NEPHRITIS}

S. Goma, M. Abdelaziz, E. El-Hakeim, M. El Zohri, S. Sayed. Assiut University, Egypt, Assiut, Egypt

Background: Systemic lupus erythematosus (SLE) is a chronic autoimmune inflammatory disease (1), characterized by the production of autoantibodies, and formation of immune complexes due to the polyclonal activation of $T$ and $B$ lymphocytes that result in tissue and organ damage (2). During inflammation, neutrophils and macrophages release serine proteases to cleave progranulin (PGRN) into granulin (GRN), which exert their pro-inflammatory effects that counteract the anti-inflammatory effects of intact PGRN (3). Insulin-like growth factor-2 (ILGF-2) binds to insulin-like growth factors (IGFs) with high affinity (4). Although reports suggest that IGFBP-2 is a reliable biomarker of renal deterioration, it is still needed to confirm that it has high sensitivity and specificity in discriminating kidney disease caused by SLE from other origins.

Objectives: The aim of this study was to explore whether PGRN and ILGF-2 can be used as useful markers not only for accurate diagnosis of patients with active lupus nephritis (LN) but also for prediction of the disease activity in these patients. Methods: Twenty-five patients with systemic lupus erythematosus, twenty-five patients with chronic renal failure and twenty-five age- and sex-matched healthy volunteers were enrolled in the study. Routine laboratory investigations and measurement of serum PRGN and IGFBP-2 levels were done.

Results: Our results showed that the mean age of SLE, CRF and control groups $31.12 \pm 12.34,38.7 \pm 9.4$ and $32.96 \pm 13.66$ respectively with no significant difference between the three groups. There was female predominance in the three groups. Disease duration was $4.78 \pm 4.26$ in SLE patients. The mean of SLEDAI score was $15.04 \pm 7.54$. All renal biopsy results were class 2,3 , and 5 with a percentage of $32 \%, 24 \%$, and $44 \%$ respectively.

Table 1. Levels of PRGN and ILGF-2 in SLE, CRF and control groups

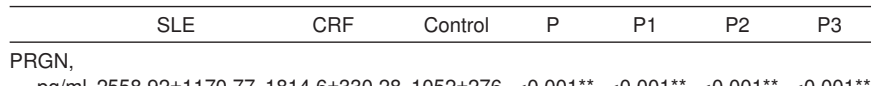

$\mathrm{pg} / \mathrm{m}$

IIGF-2,

$\mathrm{ng} / \mathrm{ml} \quad 26.44 \pm 11.55 \quad 6.14 \pm 2.25 \quad 3.3 \pm 1.7 \quad<0.001^{\star *}<0.001^{\star *}<0.001^{\star *} \quad 0.148$

P: Comparison between all groups. P1: comparison between SLE and CRF. P2: comparison between SLE and control. P3: comparison between CRF and control. Table 2. Levels of PRGN

and ILGF-2 in relation to SLEDAI score, renal SLEDAI, and Renal biopsy

\begin{tabular}{lcc}
\hline & PRGN $\mathrm{pg} / \mathrm{ml}$ & ILGF-2 $\mathrm{ng} / \mathrm{ml}$ \\
\hline SLEDAl score & & \\
$\quad$ Moderate & $1945.6 \pm 300$ & $26.8 \pm 11.7$ \\
High & $2072.1 \pm 545.5$ & $20.1 \pm 5$ \\
Very high & $4269.2 \pm 1106.8$ & $37.6 \pm 12.6$ \\
P value & $0.002^{\star \star}$ & $0.006^{\star *}$ \\
Renal SLEDAI & & \\
Inactive & $2251.2 \pm 841$ & $23.3 \pm 8.9$ \\
Active & $2764.1 \pm 1335$ & $28.6 \pm 12.9$ \\
P value & 0.311 & 0.196 \\
Renal biopsy & & \\
Class 2 & $2199.31 \pm 854.85$ & $24.38 \pm 13.31$ \\
Class 3 & $2244.17 \pm 767.97$ & $23.75 \pm 7.01$ \\
Class 4 & $2992.14 \pm 1453.93$ & $29.41 \pm 12.41$ \\
P value & 0.270 & 0.540 \\
\hline
\end{tabular}

SLEDAI: Systemic Lupus Erythematosus Disease Activity Index. *Statistically significant difference $(p<0.05){ }^{\star *}$ Statistically significant difference $(p<0.01)$

Conclusions: PGRN and ILGF-2 are significantly elevated in SLE than CRF and control and were associated with SLEDAI. Hence they are considered specific to LN.

References:

[1] Rahman A, Isenberg DA. (2008) Systemic lupus erythematosus. N Engl J Med 358:929-939.

[2] Kotzin BL. (1996) Systemic lupus erythematosus. Cell 85: 303-306.

[3] Park B, Buti L, Lee S et al. (2011) Granulin is a soluble cofactor for toll-like receptor 9 signaling. Immunity 34(4):505-513.

[4] Firth SM, Baxter RC. (2002) Cellular actions of the insulin-like growth factor binding proteins. Endocr Rev 23(6):824-54. 